\title{
CONCEPTO DE DESARROLLO HUMANO: CARITAS IN VERITATE Y PROGRAMA DE DESARROLLO DE LAS NACIONES UNIDAS
}

Martha Arellano Cruz*

\begin{abstract}
RESUMEN
Este artículo trata de dar a conocer algunos aspectos importantes del desarrollo humano integral, desde la perspectiva de las ciencias sociales, respondiendo a las preguntas: ¿Por qué y para qué es importante conocer el concepto?, ¿Por qué continúa el hambre y la pobreza en el mundo? Para dar respuesta a las interrogantes se ha considerado describir las formulaciones del desarrollo humano integral en dos partes: la primera trata de cómo es abordado el concepto en la encíclica Caritas in Veritate; la segunda, presenta los aportes del PNUD al desarrollo.
\end{abstract}

\section{PALABRAS CLAVE:}

Desarrollo Humano, PNUD, Derechos Humanos, Metas del Milenio, Bienestar Social.

\section{THE CONCEPT OF HUMAN DEVELOPMENT: "CARITAS IN VERITATE" AND THE UNITED NATIONS DEVELOPMENT PROGRAMME}

\begin{abstract}
This paper tries to put on the table some important aspects -from the perspective of social sciences -on comprehensive human development by trying to answer the following questions: Why and for what is important to under stand such concept? Why want and hunger continues to strike around the world? To answer these questions, the interpretations on comprehensive human development have been described in two parts: the first is how Caritas in Veritate understands this concept, and the second are the UNDP contributions to development.
\end{abstract}

\section{KEY WORDS:}

Human Development, UNDP, Human Rights, Millennium Development Goals, Social Welfare.

\footnotetext{
* Licenciada y Magíster en Sociología por la Pontificia Universidad Católica del Perú. Cuenta con estudios doctorales por la UNED- España. Profesora adscrita al Departamento de Filosofía y Teología de la Universidad Femenina, teniendo a su cargo la asignatura de Realidad Nacional. Se desempeña como Jefe del Área de Proyección Social en la UNIFÉ.
} 


\section{INTRODUCCIÓN}

E n la actualidad el concepto de desarrollo humano integral es muy importante para garantizar el sustento de la población mundial y que pueda vivir en buenas condiciones de vida, en paz, en democracia. Por su importancia este concepto es usado por políticos, por organizaciones no gubernamentales, el Banco Mundial y el Fondo Monetario Internacional, las Naciones Unidas, por el Papa Benedicto XVI; entre otros.

La formulación del concepto de desarrollo humano integral, al mismo tiempo que moderniza la concepción de desarrollo innova la forma y la percepción del cómo enfrentarlo, así se presenta en la encíclica Caritas in Veritate y en el contexto del Programa de Desarrollo de la Naciones Unidas (PNUD).

\section{Desarrollo humano en la encíclica Caritas in Veritate}

En la encíclica la palabra "desarrollo" aparece 258 veces en 127 páginas pequeñas, un promedio de dos veces por página. Se trata de un desarrollo humanista: Desarrollo de todos y cada uno de los hombres, de manera integral. Considera al desarrollo como el bienestar social a la solución adecuada de los problemas socioeconómicos que afligen a la humanidad. El desarrollo humano integral es el concepto fundamental en la encíclica, para ampliarlo utiliza el concepto de dignidad humana.

En el capítulo segundo el Papa Benedicto XVI (2009) toma del Papa Pablo VI (1967) la visión articulada de desarrollo. 'Con el término 'desarrollo' quiso indicar ante todo el objetivo que los pueblos salieran del hambre, la miseria, las enfermedades endémicas y el analfabetismo. Desde el punto de vista económico, eso significaba su participación activa y en condiciones de igualdad en el proceso económico internacional; desde el punto de vista social, su evolución hacia sociedades solidarias y con buen nivel de formación; desde el punto de vista político, la consolidación de regímenes democráticos capaces de asegurar libertad y paz" (CV.21).

El Papa Benedicto XVI ve con preocupación, después de muchos años, de 1967 al 2009, la relación entre desarrollo y crisis de las últimas décadas. Por lo tanto, cabe la pregunta: ¿se ha cumplido las expectativas del Papa Pablo VI en cuanto al desarrollo humano? Además, considera que es preocupación de la Iglesia por la capacidad del hombre expresada en la transformación de la naturaleza mediante el trabajo, principalmente teniendo en cuenta lo tecnológico, buscando precisar objetivos realistas y poder gestionar constante y adecuadamente los instrumentos disponibles. La ganancia es útil como medio, pero cuando el logro del beneficio se obtiene mal y sin tener en cuenta el bien común como fin último, corre el riesgo de destruir riqueza y crear pobreza.

Según el Papa Benedicto XVI, el desarrollo económico que el Papa Pablo VI proponía era un crecimiento real para todos y concretamente sostenible, en cambio tenemos que el desarrollo ha sido y sigue siendo positivo que ha sacado a muchas personas de la miseria y que, últimamente, muchos países tienen la oportunidad de participar efectivamente en la política internacional. Pero, el desarrollo económico esta originando desviaciones y problemas dramáticos y que la crisis actual lo ha intensificado.

"Es necesario reflexionar sobre las medidas necesarias para solucionar problemas que no sólo son nuevos respecto a los afrontados por el Papa Pablo VI, sino también, y sobre todo, que tienen un efecto decisivo para el bien presente y futuro de la humanidad. Los aspectos de la crisis y sus soluciones, así como la posibilidad de un futuro 
nuevo desarrollo, están cada vez más interrelacionados, se implican recíprocamente, requiere nuevos esfuerzos de comprensión unitaria y una nueva síntesis humanista. ... La crisis nos obliga a revisar nuestro camino a darnos nuevas reglas y a encontrar nuevas formas de compromiso, a apoyarnos en las experiencias positivas y a rechazar las negativas. De este modo, la crisis se convierte en ocasión de discernir y proyectar de un modo nuevo" (CV. 21). Se trata de tener en cuenta las dificultades del presente, de forma confiada y no de resignada.

Continua Benedicto XVI aludiendo que el desarrollo se manifiesta en diferentes ámbitos y que las culpas y los méritos son muchos y diferentes; deberíamos liberarnos de las ideologías que simplifican la realidad y examinar con objetividad la dimensión humana de los problemas. En los países ricos nuevos estratos sociales se empobrecen y nace nueva pobreza; en los más pobres existen categorías sociales que poseen grandes límites de riqueza que contrasta con situaciones de extrema pobreza. Esta situación es inaceptable. Se da el caso de la desigualdad distributiva de riqueza y de ingresos. Hay corrupción e ilegalidad en los actores económicos y políticos de los países del primer y tercer mundo. No se respeta los derechos humanos de los trabajadores, especialmente por las trasnacionales. Los objetivos de las ayudas del sistema financiero internacional se han desviado por la falta de responsabilidad de los países donantes y beneficiarios.

También, se manifiesta la misma articulación de responsabilidades en las causas inmateriales o culturales del desarrollo y subdesarrollo. Los países ricos, emplean la protección del derecho a la propiedad intelectual de desarrollo científico, en especial en el campo de la salud. Los países pobres mantienen formas culturales y normas sociales de comportamiento que frena el proceso del desarrollo.

\subsection{Modelo de desarrollo económico y tecnológico}

Actualmente existen sociedades que se han desarrollado, a pesar que al interior de ellas aún prevalecen grupos que viven en condiciones de pobreza extrema y hambre, y se ubican en las grandes potencias con un rol importante en el futuro.

No basta crecer desde el aspecto económico y tecnológico, salir del atraso económico; el desarrollo debe ser auténtico e integral, debe solucionar la problemática compleja de la promoción del hombre tanto en las sociedades desarrolladas y en vías del desarrollo, para salir de la explotación y de las consecuencias negativas que se derivan del crecimiento por desviaciones y desequilibrios.

Para el Papa Benedicto XVI, el replanteamiento total al modelo de desarrollo económico - tecnológico se hubiera manifestado tras la caída del sistema económico y político comunista de los países de Europa Oriental y el fin de la "Guerra Fría" o de los "Bloques contrapuestos". Para sustentar esta idea recurre a Papa Juan Pablo II, 1987, "la existencia de estos 'bloques' era una de las principales causas del subdesarrollo, pues la política sustraía recursos a la economía y a la cultura, y la ideología inhibía la libertad. En 1991, después de los acontecimientos de 1989, pidió también que el fin de los bloques se correspondiera con un nuevo modo de proyectar globalmente el desarrollo, no sólo en aquellos países, sino también en occidente y en las partes del mundo que se estaba desarrollando" (CV. 23).

En ese momento histórico la "cuestión social" mundial estaba menos integrada y menos interdependiente que la actual. La actividad productiva se realizaba en el ámbito nacional y las inversiones financieras no tenían grandes vínculos con el sistema financiero internacional. La política de muchos Estados - Nación podían fijar 
sus prioridades económicas y gobernar su dirección.

\subsection{Desarrollo humano integral}

Actualmente, los Estados - Nación están limitados por el nuevo contexto económico comercial y financiero internacional. El Papa Benedicto XVI plantea la alternativa al modelo de desarrollo económico - tecnológico: "Hoy, aprendiendo también la lección que proviene de la crisis económica actual, en la que los poderes públicos del Estado se ven llamado a corregir errores y disfunciones, parece más realista una renovada valoración de su papel y de su poder, que han de ser sabiamente reexaminados y revalorizados, de modo que sean capaces de afrontar los desafíos del mundo actual, incluso con nuevas modalidades de ejercerlos. Con un papel mejor ponderado de los poderes públicos, es previsible que se fortalezcan las nuevas formas de participación en la política nacional e internacional que tiene lugar a través de la actuación de las organizaciones de las sociedad civil; en este sentido, es de desear que haya mayor atención y participación en la res pública por parte de los ciudadanos" (CV. 24).

El Papa Benedicto XVI, sostiene que a muchos países les cuesta trabajo lograr sus objetivos de verdadera justicia social en el marco de la transformación social. El mercado global tiene como fin, en especial los países del primer mundo, buscar áreas para producir a bajo costo, reducir precios de los bienes y servicios ${ }^{30}$ y acelerar el índice de crecimiento, creando un mercado de mayor consumo en el interior del país. Por los cambios sociales y económicos las organizaciones sindicales presentan mayores dificultades para desarrollar su representación de los intereses de los asociados; los gobiernos por razones económicas, cada vez, están limitando las libertades sindicales.
La movilidad laboral ha estimulado la producción de la nueva riqueza y el intercambio entre culturas diferentes. Sin embargo, la movilidad también trae consigo la incertidumbre de las condiciones de trabajo, generando inestabilidad psicológica en la organización social, incluido el matrimonio. Se debe considerar "el primer capital que se ha de salvaguardar y valorar es el hombre, la persona en su integridad" (CV. 25).

En la actualidad, el diálogo intercultural es más frecuente y para ser eficaz tiene como punto de partida una toma de conciencia de la identidad de los que están dialogando. El diálogo dado a la mercantilización de los intercambios culturales, tiene un doble riesgo. En primer lugar, un eclecticismo cultural: alude a que las culturas son superpuestas unas a otras. En segundo lugar, rebajar la cultura y homologar los comportamientos y estilos de vida. Ambos riesgos separan la cultura de la naturaleza humana. No debe llegar a la situación en que la humanidad corra el riego de sometimiento y manipulación.

Con la extrema inseguridad alimentaria: los países pobres llegan al hambre, que depende de la escasez material, insuficiencia de recursos sociales e institucionales. Es decir, la falta de instituciones que garanticen el acceso, de las familias que viven en condiciones de pobreza extrema, al agua y a la comida diaria, y con valor nutricional. El derecho a la alimentación y al agua es importante para conseguir otros derechos, especialmente a la vida.

Es importante tener en cuenta, además, "que la vía solidaria hacia el desarrollo de los países pobres puede ser un proyecto de solución de la crisis global actual, como lo han intuido en los últimos tiempos hombres políticos y responsables de instituciones internacionales (CV. 27). Uno de

30 Este proceso ha llevado a la reducción de la red de seguridad social a cambio de búsqueda de mayores ventajas competitivas en el mercado global. (Benedicto XVI Caritas in Veritate p. 33) 
los aspectos más destacados del desarrollo actual es la importancia del tema respeto a la vida, que en modo alguno debe separase de las cuestiones relacionadas con el desarrollo de los pueblos. Es un aspecto que últimamente está asumiendo cada vez mayor relieve, obligándonos a ampliar el concepto de pobreza y de subdesarrollo a los problemas vinculados con la acogida de la vida sobre todo donde está se ve impedida de diversas formas"... "La apertura a la vida está en el centro del verdadero desarrollo" (CV. 28). El desarrollo tiene otro aspecto de la vida que se interrelaciona muy estrechamente y que en la actualidad se presenta: la negación del derecho a la libertad religiosa. Luchas y conflictos que se producen entre sociedades por motivos religiosos, con el afán de poder y riqueza. "Hoy se mata frecuentemente en nombre sagrado de Dios" (CV. 29).

El desarrollo humano integral es más complejo, que el desarrollo o las medidas socioeconómicas: la correlación entre sus múltiples elementos exige un esfuerzo para que los diferentes ámbitos del saber sean interactivo, con vistas de un verdadero desarrollo de los pueblos".... El saber humano es insuficiente y las conclusiones de las ciencias no podrán indicar por sí solas la vía hacia el desarrollo integral del hombre, siempre hay que lanzarse más allá: lo exige la caridad en la verdad" (CV. 30). "El desarrollo es imposible sin hombres rectos, sin operadores económicos y agentes políticos que sientan fuertemente en su conciencia la llamada al bien común" (CV. 71).

\section{Una perspectiva del desarrollo hu- mano, desde el PNUD - Naciones Unidas}

Frente a la inquietud que mostró el mundo en la década de los noventa, que se constató que el crecimiento de la producción no iba con el crecimiento del empleo y mejora de ingresos, se plantea el paradigma del desarrollo humano que postula la necesidad de establecer nuevas relaciones entre el Estado y el mercado como fuerzas complementarias que podrían resolver el problema de desempleo y subempleo. Esta perspectiva busca la combinación entre la eficiencia económica individual y la solidaridad social para atender a la población excluida del mercado.

El paradigma esta relacionado con el concepto de seguridad humana: protección a las personas en su soberanía o integridad física, y la aceptación de la preservación de derechos, valores y culturas. La seguridad humana amenazada en cualquier parte del mundo, implica y compromete a todas las personas del mundo. La preocupación por las hambrunas, los conflictos étnicos, la desintegración social, el terrorismo, la contaminación, el tráfico de drogas ya no pueden limitarse a las fronteras nacionales. La seguridad humana es un concepto globalizado y es por ello que están surgiendo nuevos retos mundiales para su preservación.

El primer informe sobre Desarrollo del PNUD (1990) contribuyó a ubicar al ser humano en el centro del desarrollo, ha proporcionado un replanteo sobre el progreso humano a escala mundial y de las diferentes estrategias que aplican los países para lograr el bienestar humano. A partir de 1995 cada año el PNUD presenta un Informe sobre Desarrollo Humano, enfatizando sobre algún tema que sirve para profundizar el concepto. Este es asumido como: "un proceso conducente a la ampliación de las opciones de que disponen las personas. En principio esas opciones pueden ser infinitas y pueden cambiar a lo largo del tiempo. Pero a todos los niveles de desarrollo, las tres opciones esenciales para las personas son: poder tener una vida larga y saludable, poder adquirir conocimientos y poder tener acceso a los recursos necesarios para disfrutar de un nivel de vida decoroso. Si no se dispone de estas opciones esenciales muchas otras oportunidades permanecen inaccesibles" (PNUD, 1995). 
Otras opciones son importantes para el Desarrollo Humano, van desde la libertad política, económica y sociocultural hasta las oportunidades de ser creativos y productivos y de disfrutar de autorrespeto personal y de derechos humanos garantizados. Por consiguiente el desarrollo humano tiene dos fases. Una es el fenómeno de la capacidad humana, como mejor salud y mayores conocimientos teóricos y prácticos. La otra, es el aprovechamiento de la capacidad adquirida por las personas con fines productivos o de creación, o en actividades culturales, sociales y políticas. Si no se equilibran estas fases, puede ocasionar una gran frustración humana.

En el desarrollo humano el ingreso es sólo una opción a las que aspiran las personas, pero, no abarca el total de sus vidas. El propósito del desarrollo es ampliar todas las opciones humanas y no sólo el ingreso. Por lo tanto, se separa de las teorías y modelos de crecimiento económico que refieren específicamente el aumento del Producto Bruto Interno (PBI), en lugar de mejorar la calidad de la vida humana.

El enfoque de bienestar social considera a los seres humanos como beneficiarios y no como agentes de cambio en el proceso de desarrollo. El enfoque de necesidades básicas se centra en proporcionar bienes y servicios materiales a grupos de población que padecen privaciones, en lugar de ampliar las opciones humanas en todas las esferas. El concepto de desarrollo humano incluye los enfoques mencionados, pero los supera, analiza las cuestiones sociales - crecimiento económico, el comercio, el empleo, la libertad política o los valores culturales - desde la perspectiva del ser humano. Se centra en ampliar las opciones humanas y se aplica por igual a países en desarrollo e industrializados.
Hay en el concepto de desarrollo humano cuatro elementos principales: productividad, equidad, sostenibilidad y potenciación. Éstos son importantes mientras que las mujeres se les incluya en proceso de desarrollo, caso contario el desarrollo seguirá siendo débil y desnivelado. Dados los adelantos humanos en algunas categorías sociales y sociedades, también existen retrocesos en otras. Podemos considerar las siguientes situaciones:

1. Las sociedades en desarrollo han experimentado en los últimos 30 años adelantos en materia de desarrollo humano. Pero, las disparidades entre los países del Norte y del Sur se mantienen.

2. A pesar del progreso, en los países industrializados y en desarrollo subsisten considerables privaciones. En los países en desarrollo, una de cada tres personas vive en condiciones de pobreza. En los países industrializados, hay cerca de 100 millones de personas por debajo de la línea de la pobreza. Muchas personas en ambas sociedades se sienten amenazadas por lo que representa los estupefacientes, la carencia de vivienda, el desempleo, la contaminación, el SIDA y el delito.

3. Es posible la cooperación para el desarrollo centrando las energías nacionales e internacionales. Pero, a menudo se presenta la excusa de la escasez del recurso financiero.

4. Los retos fundamentales de desarrollo humano para el siglo XXI requieren de celebraciones de pactos mundiales.

El informe sobre desarrollo humano (2000) manifiesta que los derechos humanos y el desarrollo humano tienen una visión y un propósito común: velar 
por la libertad, el bienestar y la dignidad de todos. Uno de los logros del siglo XX es el progreso en derechos humanos. En 1900 más de la mitad de la población vivía en régimen colonial, hoy en día alrededor de tres cuartas partes del mundo vive en regímenes democráticos. Además, ha habido gran progreso en la eliminación de la discriminación en cuanto a la raza, la religión y el género, y en el derecho a la escolaridad y a la atención básica de la salud, progreso que podemos decir aún se mantiene.

El informe sobre desarrollo humano (2002) alude que el concepto de desarrollo humano, como proceso y como fin, distingue entre la formación de las capacidades humanas y la manera cómo los individuos emplean las capacidades adquiridas. La primera alude a la salud y al conocimiento; la segunda al trabajo, a la creación y al descanso. En este enfoque la ampliación de las capacidades se da por la ampliación de las posibilidades de acción y de las diferentes formas de ser que tienen a su alcance las personas con libertad para seleccionar las capacidades para desempeñarse en la vida. La promoción del desarrollo humano requiere de una gobernabilidad democrática, de gozar de una libertad política y poder participar en las decisiones que afectan a los individuos y exigir políticas que amplíen las oportunidades sociales, económicas y culturales.

El informe sobre el desarrollo humano (2006), plantea que este concepto se retroalimenta de la política, que implica democracia y libertad con fines concretos, componentes éticos asociados y con procesos descentralistas. La democracia no puede ser centralista, y la descentralización debe ser democrática.

El concepto de desarrollo humano es más profundo y rico que cualquier índice o de cualquier indicador estadístico. Pero, necesita de un indicador para abordar en forma simplificada una realidad compleja, el PNUD presenta el Índice de Desarrollo Humano (IDH), posee tres componentes: esperanza de vida al nacer, nivel educacional integrado por la alfabetización de adultos y tasa combinada de matricula primaria, secundaria y terciaria e ingreso. El valor del IDH para cada país indica cuánto avanzado en llegar a ciertas metas de seguridad. Cuánto más cercano esté un país a un IDH igual a 1, menor será la distancia que debe recorrer; en esta condición se encuentran los países industrializados, caso contrario ocurre con los países en desarrollo.

El desarrollo humano no comienza como un modelo predeterminado. Se inspira en las metas de largo plazo de una sociedad y hace radicar sus principios en una visión humanista.

\subsection{Objetivos del Desarrollo del Mi- lenio}

En el año 2000 los líderes del mundo establecieron 8 objetivos, 18 metas y 48 indicadores a largo plazo, para liberar a una gran parte de la humanidad de la pobreza extrema, el hambre, el analfabetismo y las enfermedades, igualdad de sexos y el empoderamiento de la mujer, la sostenibilidad del medio ambiente y una alianza mundial para el desarrollo. Es decir, aprobaron un plan para un mundo mejor y prometieron establecer esfuerzos en la materialización de la visión.

Los ocho Objetivos del Desarrollo del Milenio (ODM) (2008) son: 1. Erradicar la pobreza extrema y el hambre; 2. Lograr la educación primaria universal; 3. Promover la igualdad de género y la autonomía de la mujer; 4. Reducir la mortalidad de los niños menores de cinco años; 5. Mejorar la salud materna; 6. Combatir el VIH/SIDA, 
el paludismo y otras enfermedades; 7 . Garantizar la sostenibilidad del medio ambiente; 8. Fomentar una alianza mundial para el desarrollo.

La Declaración de Milenio (2000) fijó el año 2015 como fecha límite para alcanzar la mayoría de los ODM, establecieron los parámetros cuantitativos para reducir todas las formas de extrema pobreza y el hambre a la mitad. Pero, a medida que se acerca la fecha, falta cinco años, el mundo se encuentra en una crisis económica sin precedentes por su gravedad y sus dimensiones mundiales.

Según el Informe Objetivos del Desarrollo del Milenio 2009, los avances hacia el logro de los ODM se ven amenazados por el crecimiento económico lento e incluso negativo, una disminución de recursos, menos oportunidades comerciales para los países en desarrollo y posibles reducciones de los fondos provenientes de países donantes. Además, los efectos del cambio climático son cada vez más evidentes y pueden tener un impacto devastador tanto en los países ricos y pobres. Las relaciones de las alianzas mundiales deben guiar acciones colectivas.

No se podrá cumplir con las metas de los ODM, lo que sumiría que miles de millones de personas más en la pobreza y elevaría el riesgo de disturbios sociales y políticos, que se debe evitar. Esta situación se considera como una oportunidad para tomar decisiones difíciles para crear un futuro más equitativo y sostenible. Es el momento de acelerar los avances hacia el logro de los ODM y fortalecer la alianza mundial para el desarrollo. Si la comunidad mundial responde de manera constructiva a la crisis, los objetivos podrían lograrse. Es importante tener en cuenta que los intereses de los países pobres sigan siendo el eje central en las negociaciones comerciales.
Los importantes avances en la lucha contra la pobreza extrema entre $1990 \mathrm{y} \mathrm{el}$ 2005 se han estancado. En el año 2009, se estimó que entre 55 a 90 millones de personas, más de lo previsto antes de la crisis, estarán viviendo en condiciones de pobreza extrema. Además, la prevalencia del hambre en las regiones en desarrollo está aumentando y ha pasado de un 16\% a un $17 \%$ en el año 2008. Asimismo, más de la cuarta parte de los niños tienen insuficiencia ponderal para su edad, limitando sus perspectivas de supervivencia, crecimiento y desarrollo a largo plazo.

Los Jefes de Estado y de Gobierno reunidos en la Sede de la Naciones Unidas en Nueva York del 20 al 22 de septiembre de 2010 manifestaron su profunda preocupación porque aún están lejos de cubrir las necesidades. Recordaron los objetivos y compromisos de desarrollo derivados de la Declaración del Milenio y el Documento Final de la Cumbre Mundial 2005, reafirmaron la determinación de trabajar juntos para promover el adelanto económico y social de todos los pueblos.

Reconocieron que los países en desarrollo han realizado considerables esfuerzos en pro del cumplimiento de los Objetivos de Desarrollo del Milenio y han tenido buenos logros, alcanzando algunas metas; pero también reconocieron que hay mucho por hacer para lograr los ODM, ya que el progreso ha sido desigual de una región a otra y entre países y dentro de ellos. El hambre y la mal nutrición aumentaron de nuevo entre 2007 y 2009, menoscabando parcialmente los logros alcanzados hasta entonces. "Se ha progresado lentamente en el logro del empleo pleno y productivo y el trabajo decente para todos, el adelanto de la igualdad entre los géneros y el empoderamiento de la mujer, el logro de la sostenibilidad ambiental y el suministro de saneamiento básico, y las nuevas infecciones de VIH todavía supera el número de 
personas que inician el tratamiento. En particular, expresamos nuestra grave preocupación por el lento progreso en reducir la mortalidad materna y mejorar la salud materna y reproductiva. Los progresos en otros objetivos de Desarrollo del Milenio son escasos $y$ deben mantenerse para evitar que se echen a perder". ${ }^{31}$

Subrayaron el rol fundamental de la alianza mundial para el desarrollo y la importancia del octavo objetivo para lograr los Objetivos de Desarrollo del Milenio. Reconocieron que sin el apoyo internacional, muchos países en desarrollo no podrán alcanzar los objetivos para el 2015.

Mostraron además su preocupación por el impacto de la crisis financiera y económica, la más grave desde la Gran Depresión. Ha echado a perder algunos logros alcanzados en muchos países en desarrollo y amenaza con no llegar a las metas de los Objetivos de Desarrollo del Milenio par el año 2015.

\section{CONCLUSIONES}

El modelo de desarrollo humano descrito en la Encíclica Caritas in Veritate y de las Naciones Unidades manifiestan que debe ser utilizado para dar solución a los problemas de marginalidad social, económica, política, cultural, religiosa; en especial a la situación de pobreza, pobreza extrema y de hambre.

En algunos países habido progreso en el desarrollo humano, en cambio en otros aún sigue la situación de exclusión social, donde las personas no tienen acceso a las oportunidades del desarrollo.
El problema para alcanzar niveles altos de desarrollo se ve afectada por la crisis financiera y económica mundial, especialmente en las relaciones de cooperación internacional, los países desarrollados han dejado de brindar financiamiento, por lo tanto, los países en vía de desarrollo no podrán alcanzar el bien común, la paz, y la felicidad de sus pobladores.

\section{REFERENCIAS}

Benedicto XVI (2009). Carta Encíclica Caritas in Veritate. Lima. Editorial Paulinas.

Naciones Unidas (1990). Informe sobre Desarrollo Humano. México, PNUD.

(1995). Informe sobre Desarrollo Humano. México, PNUD.

(2000). Informe sobre Desarrollo Humano. México, PNUD.

(2002). Informe sobre Desarrollo Humano. México, PNUD.

(2006). Informe sobre Desarrollo Humano. México, PNUD.

(2008). Objetivos de Desarrollo del Milenio. México, PNUD.

(2009). Objetivos de Desarrollo del Milenio. México, PNUD.

(2010). Cumbre Mundial sobre los objetivos de desarrollo del Milenio. Nueva York del 20 al 22 de septiembre.

31 Cumbre Mundial sobre los objetivos de desarrollo del Milenio. 2010. (pp.4 y 5). 